\title{
Optical characterization of aminosilane-modified silicon dioxide surface for biosensing
}

\section{Terracciano}

I. Rea

ilaria.rea@na.imm.cnr.it

\section{J. Politi}

\section{De Stefano}

\begin{abstract}
Institute for Microelectronics and Microsystems, National Council of Research, Via P. Castellino 111, I-80131 Naples, Italy

Dept. of Pharmacy, University of Naples Federico II, Naples, Italy

Institute for Microelectronics and Microsystems, National Council of Research, Via P. Castellino 111, I-80131 Naples, Italy

Institute for Microelectronics and Microsystems, National Council of Research, Via P. Castellino 111, I-80131 Naples, Italy

Dept. of Chemical Sciences - "Federico II" University of Naples, Naples Italy
\end{abstract}

Institute for Microelectronics and Microsystems, National Council of Research, Via P. Castellino 111, I-80131 Naples, Italy

Silicon dioxide surfaces, functionalized by two aminosilane compounds (3-amino-propyl-triethoxysilane, APTES; 3-amino-propyl-dimethylethoxysilane, APDMES) both dissolved in different solvents (dry ethanol and toluene), have been investigated by standard techniques such as spectroscopic ellipsometry (SE), water contact angle (WCA), and atomic force microscopy (AFM). Silane thicknesses between 5 and $80 \AA$ have been found, depending on deposition conditions; surface wettabilities change, accordingly. These organic-inorganic interfaces have also been modified by a cross-linker (bis-sulfosuccinimidyl suberate) in order to covalently bind a fluorescein labeled protein A. The amount of protein linked to functional surfaces has been quantified by SE and fluorescence microscopy. These results could be very useful in developing new platforms for optical biosensing.

[DOI: http://dx.doi.org/10.2971/jeos.2013.13075]

Keywords: Surface functionalization, biomaterials, ellipsometry, water contact angle, atomic force microscopy

\section{INTRODUCTION}

Bioconjugation chemistry is a key issue not only for fabrication of sensitive and selective biosensors, but also for many technological devices, which can be used in biomedical diagnostics and even in fundamental scientific studies. In the last few years, multifunctional lab-on-chip platforms for biomolecular interactions monitoring have been proposed [1]-[3], as well as new single molecule spectroscopy methodologies $[4,5]$, all based on specific passivation protocols of support surfaces. The aim of these treatments is immobilization on a solid support of biological molecules, preserving their specific functionalities through a good control of their orientation and organization. Even if glass [6] and gold [7] have been classically used, and therefore their passivation chemistries deeply studied in these kinds of application, on the other hand, silicon, and silicon related materials, are attracting growing interest, due to widespread diffusion of microfabrication technologies, well developed in the frame of consumer electronics. The most common route of silicon surfaces functionalization is to attach alkylsilanes layers through the formation of $\mathrm{Si}-\mathrm{O}-\mathrm{Si}$ bonds between the silanol groups present on oxidized silicon surface and the hydrolyzed organosilane molecules [8]. Recently, the interaction mechanism between silane layers and silicon surfaces have been deeply characterized, up to molecular level [9]-[11]. Although wet deposition by solution immersion is the most common method to prepare these samples, monofunctional and tri-functional aminosilane molecules, like 3-amino-propyl-triethoxysilane, APTES, and 3-amino-propyldimethyl-ethoxysilane, APDMES, respectively have been deposited on dehydrated silicon support also by chemical vapour deposition, which is a robust process, currently used in semiconductors industry [12]. APTES and APDMES have structural differences, as schematized in Figure 1. APTES has three attachment points to the surface or other silane molecules, therefore it can polymerize. Conversely, APDMES has only one attachment point and it cannot polymerize. In this work, we have experimentally characterized amino-

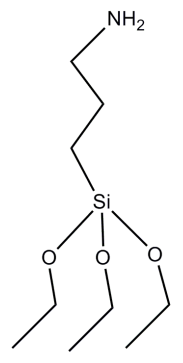

APTES

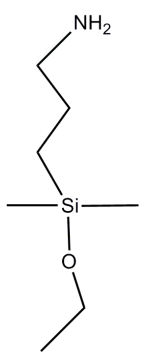

APDMES
FIG. 1 Structures of 3-aminopropyltriethoxysilane (APTES) and 3-aminopropyldimethylethoxysilane (APDMES). 


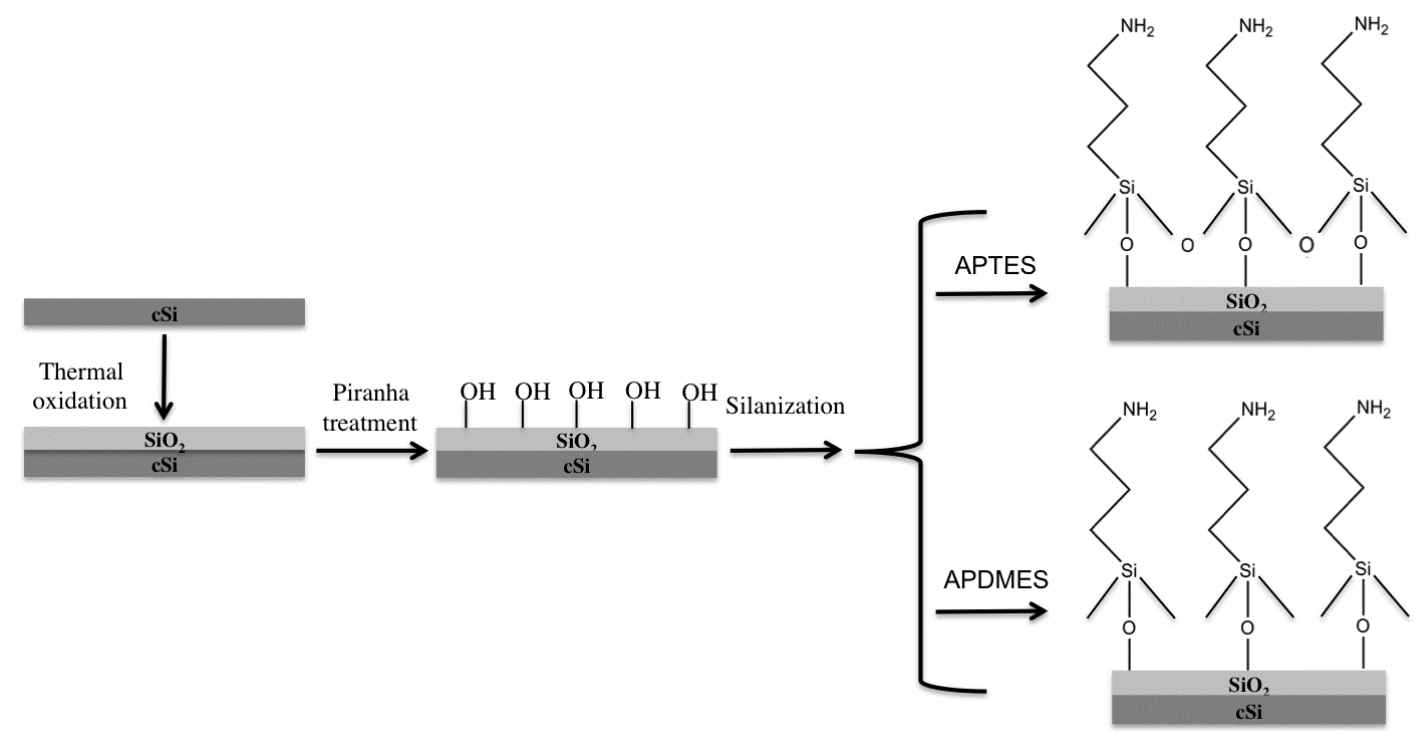

FIG. 2 Scheme of silicon surface modification by APTES and APDMES.

\begin{tabular}{|c|c|c|c|}
\hline Sample & Silane & Solvent & $\begin{array}{c}\text { Incubation time } \\
\text { (min) }\end{array}$ \\
\hline S1 & Aptes 5\% & Ethanol & 30 \\
S2 & Aptes 5\% & Ethanol & 60 \\
S3 & Aptes 5\% & Toluene & 30 \\
S4 & Aptes 5\% & Toluene & 60 \\
S5 & Apdmes 5\% & Ethanol & 30 \\
S6 & Apdmes 5\% & Ethanol & 60 \\
S7 & Apdmes 5\% & Toluene & 30 \\
S8 & Apdmes 5\% & Toluene & 60 \\
\hline
\end{tabular}

TABLE 1 Sample preparation conditions.

modified silicon dioxide surfaces by several techniques, such as spectroscopic ellipsometry (SE), water contact angle (WCA), and atomic force microscopy (AFM). Data highlight some important features of these surface passivation strategies useful in realization of immune-arrays or, in general, bioconjugated devices.

\section{MATERIAL AND METHODS}

\subsection{Silane surface modifications}

Highly doped $\mathrm{p}^{+}$silicon wafer, $<100>$ oriented, $0.003 \Omega \cdot \mathrm{cm}$ resistivity, $400 \mu \mathrm{m}$ thick, was cut into $10 \mathrm{~mm} \times 10 \mathrm{~mm}$ square pieces. After cleaning by means of standard RCA process [13], silicon substrates were thermally oxidized at $1050^{\circ} \mathrm{C}$ for 5 hours. Chips were, then, immersed in piranha solution $\left(\mathrm{H}_{2} \mathrm{SO}_{4}: \mathrm{H}_{2} \mathrm{O}_{2}=4: 1\right)$ at room temperature for $30 \mathrm{~min}$ so as to create $\mathrm{Si}-\mathrm{OH}$ groups on silicon surface, extensively washed in milli $Q$ water, and dried in a stream of nitrogen gas. Eight different silane films, namely S1-S8, were obtained by incubating the silicon substrates at room temperature, for 30 or $60 \mathrm{~min}$, into $5 \%$ silane solutions prepared by direct dissolution of silane, APTES or APDMES, in ethanol or anhydrous toluene, as summarized in Table 1. After silanization, silicon chips were rinsed three times in the solvent used for the process for $2 \mathrm{~min}$ so as to remove silane excess. The last step is silane curing on heater at $100^{\circ} \mathrm{C}$ for $10 \mathrm{~min}$. The scheme of silanization process performed on silicon surface is reported in Figure 2. The experiment has been performed on two sets of identical samples to confirm results.

\subsection{Biofunctionalization}

Chemicals and solvents were purchased from SigmaAldrich. Protein A labeled with -FITC (PrA*) was immobilized on silane modified silicon surface using bis(sulfosuccinimidyl)suberate $\left(\mathrm{BS}^{3}\right)$ crosslinker. The scheme of functionalization process is reported in Figure 3. Each chip was incubated with $150 \mu$ of $1.6 \mathrm{mM}$ $\mathrm{BS}^{3}$ in PBS solution (0.1 M; $\left.\mathrm{pH}=7.4\right)$ at $4^{\circ} \mathrm{C}$ for 5 hours. $\mathrm{N}$-hydroxysulfosuccinimide (NHS) ester reacts (through $\mathrm{SN}_{2}$ ) with primary amines of silanized surface forming stable amine bonds and releasing a NHS group. The functionalized substrate was then incubated overnight $(\mathrm{ON})$ at $4^{\circ} \mathrm{C}$ with $150 \mu \mathrm{l}$ of $2 \mathrm{mg} / \mathrm{ml} \mathrm{PrA}^{*}$ in PBS (0.1 M; pH=7.4) buffer. NHS ester reacts with primary amines in the side chain of lysine residues of $\operatorname{PrA}^{*}$ forming stable amine bonds and releasing an other one NHS group.

\subsection{Spectroscopic ellipsometry}

Spectroscopic ellipsometry (SE) measurements were performed by a Jobin Yvon UVISEL-NIR phase modulated spectroscopic ellipsometer apparatus, at an angle of incidence of $65^{\circ}$ over the range $300-1600 \mathrm{~nm}$ with a resolution of $5 \mathrm{~nm}$. The instrument measures the spectral variation of the ellipsometric angles $\Psi$ and $\Delta$ defined through the relation:

$$
\operatorname{tg} \Psi e^{i \Delta}=\frac{R_{p}}{R_{S}}
$$

where $R_{p}$ and $R_{s}$ are the complex reflection coefficients of the light polarized parallel and perpendicular to plane of incidence. Thickness of films present on silicon surface was determined from the ellipsometric data analysis using Delta Psi software [14]. 

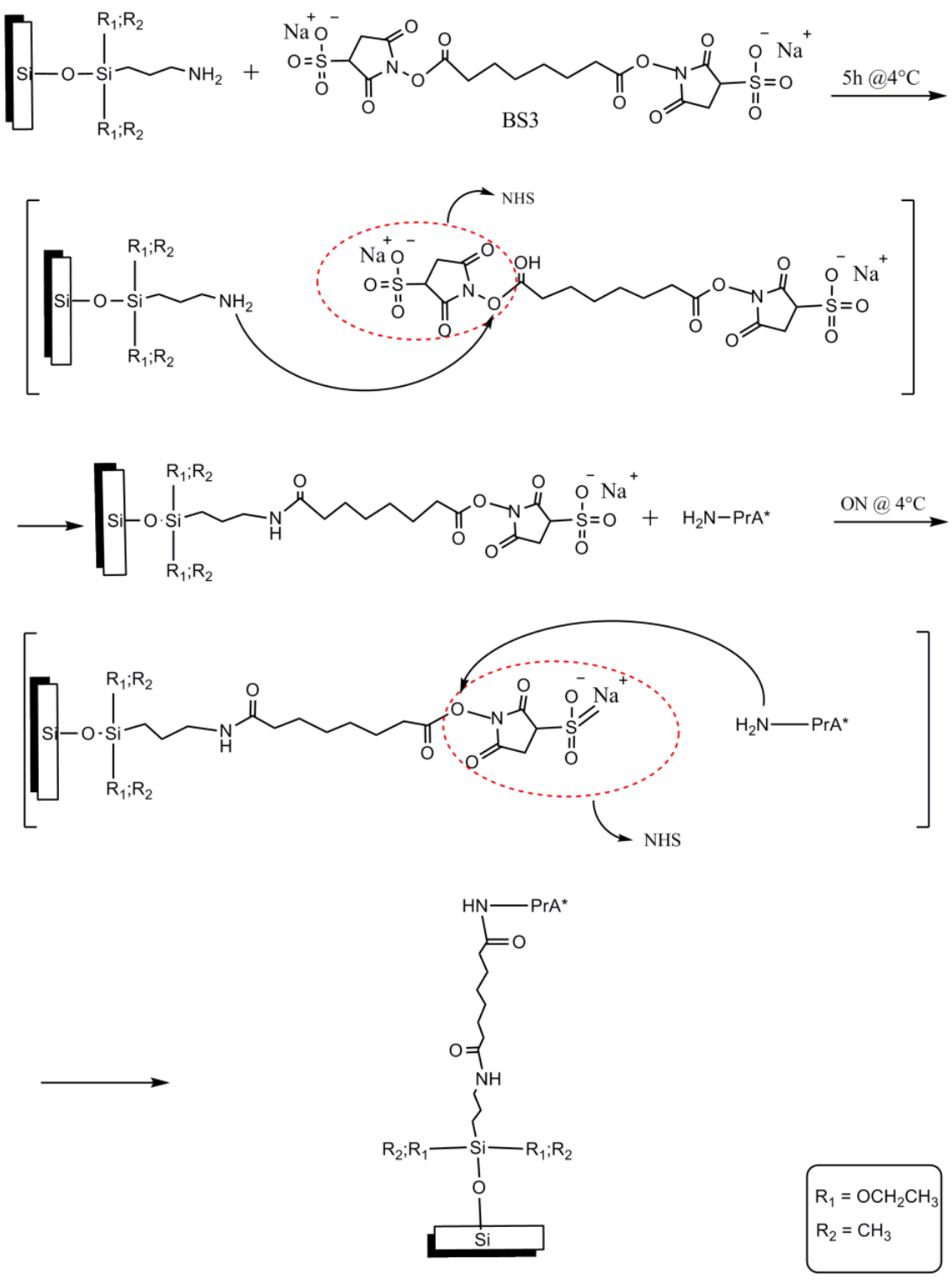

FIG. 3 Functionalization of silane modified cSi surface with PrA*.

\subsection{Water contact angle measurements}

Sessile drop technique has been used for water contact angle (WCA) measurements on a First Ten Angstroms FTA 1000 C Class coupled with drop shape analysis software. The WCA values reported in this work are the average of at least three measurements.

\subsection{Atomic Force Microscopy}

A XE-100 AFM (Park Systems) was used for the imaging of biofilms. Surface imaging was obtained in non-contact mode using Silicon/aluminum coated cantilevers (PPP-NCHR 10M; Park Systems) $125 \mu \mathrm{m}$ long with a resonance frequency of 200 to $400 \mathrm{kHz}$ and nominal force constant of $42 \mathrm{~N} / \mathrm{m}$. The scan frequency was typically $1 \mathrm{~Hz}$ per line. Roughness has been calculated on $3 \mu \mathrm{m} \times 3 \mu \mathrm{m}$ images.

\subsection{Fluorescence microscopy}

Fluorescence analysis was performed by means of a Leica Z16 APO fluorescence macroscope equipped with a camera Leica
DFC300. I3 filter was used for image acquisition consists in a 450-490 $\mathrm{nm}$ band-pass excitation filter, a $510 \mathrm{~nm}$ dichromatic mirror, and a $515 \mathrm{~nm}$ suppression filter. Fluorescence intensity values reported in the work are averaged on three measurements.

\section{RESULTS AND DISCUSSION}

A precise estimation of silane layers thickness requires the ellipsometric analysis of samples before and after silanization processes. The measure performed on bare silicon dioxide (before silanization) allows an exact determination of its thickness $(95.46 \pm 0.03 \mathrm{~nm})$ and a consequence certain estimation of silane layer. Refractive index of silicon dioxide as function of wavelength is taken from reference [15].

Spectroscopic ellipsometry data, reported in Table 2 together with water contact angle variations (i.e., the difference between the WCA of sample after and before silanization process. WCA of bare silicon dioxide surface is $\left.(60.8 \pm 0.8)^{\circ}\right)$, reveal a common trend for all characterized samples: the esti- 


\begin{tabular}{|c|c|c|c|c|c|}
\hline Silane & $n_{\text {silane }}$ & $\begin{array}{c}d \\
\left(\mathbf{g} / \mathbf{c m}^{3}\right)\end{array}$ & $\begin{array}{c}\boldsymbol{M} \\
(\mathbf{g} / \mathbf{m o l})\end{array}$ & $\begin{array}{c}\mathbf{V}_{\mathbf{m}} \\
\left(\mathbf{c m}^{3} / \mathbf{m o l}\right)\end{array}$ & $\begin{array}{c}A \\
\left(\mathbf{c m}^{3} / \mathbf{m o l}\right)\end{array}$ \\
\hline APTES & 1.46 & 0.946 & 221.37 & 234 & 65 \\
APDMES & 1.46 & 0.857 & 161.32 & 188 & 52 \\
\hline
\end{tabular}

TABLE 3 Ellipsometry data and increase of WCA after silanization processes.

\begin{tabular}{|c|c|c|}
\hline Sample & $\begin{array}{c}\text { Silane thickness } \\
(\mathbf{\AA})\end{array}$ & $\begin{array}{c}\Delta \mathbf{W C A} \\
(\mathbf{})\end{array}$ \\
\hline S1 & $5.5 \pm 0.3$ & $3 \pm 1$ \\
S2 & $17.3 \pm 0.3$ & $12.3 \pm 0.8$ \\
S3 & $26.4 \pm 0.3$ & $7.2 \pm 0.6$ \\
S4 & $81.3 \pm 0.8$ & $21 \pm 3$ \\
S5 & $0.2 \pm 0.2$ & $2 \pm 1$ \\
S6 & $3.0 \pm 0.3$ & $10 \pm 3$ \\
S7 & $4.2 \pm 0.3$ & $19 \pm 3$ \\
S8 & $10.6 \pm 0.3$ & $15 \pm 2$ \\
\hline
\end{tabular}

TABLE 2 Ellipsometry data and increase of WCA after silanization processes.

mated siloxanes film thickness increases with time deposition: 60 min samples are always thicker than 30 min samples in every combination of solute (APTES and APDMES) and solvent (ethanol and toluene). Moreover, APTES thin films are thicker than APDMES ones: a somehow expected result since APTES is able to reticulate, while APDMES is not. WCA measurements highlight that both APTES and APDMES surfaces can be sensitively less hydrophilic than uncovered ones. However, WCA values are scattered; this behavior can be explained considering film inhomogeneity due to the deposition by solution. Another very interesting application of spectroscopic ellipsometry is calculation of surface concentration that gives idea of how dense is the film deposited on the surface. To this aim, molar refractivity $A$ of a material can be defined by the formula [16]:

$$
A=\frac{\left(n^{2}-1\right)}{\left(n^{2}+2\right)} V_{m}
$$

where $n$ is the refractive index, and $V_{m}$ the molar volume calculated as the ratio $M / d$, with $M$ molecular weight, and $d$ density. Estimated values of molar refractivity for APTES and APDMES have been reported in Table 3 together with parameters used to calculate them; refractive indexes $n$ were determined by means of spectroscopic ellipsometry using a fixed index model (i.e., $\mathrm{n}(\lambda)=$ constant) [11]; molecular weight $M$, and density $d$ were taken from data sheet of chemical compounds.

These values were used to calculate surface concentration $\Gamma\left(\mu \mathrm{g} / \mathrm{cm}^{2}\right)$ of silane layers deposited on silicon chips, by Cuypers formula [17]:

$$
\Gamma=0.1 t \frac{M}{A} \frac{n_{f}^{2}-1}{n_{f}^{2}+2}
$$

where thickness $t$ has been calculated by SE (see values in Table 2). In Table 4 , we have reported the ratio $M / A$ used to calculate $\Gamma$, and values of $\Gamma$.

Just like film thicknesses, also the surface concentrations follow the same behavior: as longer time deposition is, greater

\begin{tabular}{|c|c|c|}
\hline Sample & $\begin{array}{c}\mathbf{M} / \mathbf{A} \\
\left(\mathbf{g} / \mathbf{c m}^{3}\right)\end{array}$ & $\begin{array}{c}\Gamma \\
\left(\mu \mathbf{g} / \mathbf{c m}^{3}\right)\end{array}$ \\
\hline S1 & 3.406 & $0.0051 \pm 0.0003$ \\
S2 & 3.406 & $0.0161 \pm 0.0003$ \\
S3 & 3.406 & $0.0246 \pm 0.0003$ \\
S4 & 3.406 & $0.0758 \pm 0.0007$ \\
S5 & 3.102 & $0.0001 \pm 0.0002$ \\
S6 & 3.102 & $0.0025 \pm 0.0003$ \\
S7 & 3.102 & $0.0036 \pm 0.0003$ \\
S8 & 3.102 & $0.0090 \pm 0.0003$ \\
\hline
\end{tabular}

TABLE 4 Surface concentration, $\Gamma$.

\begin{tabular}{|c|c|c|}
\hline Sample & $\begin{array}{c}\mathbf{B S}^{\mathbf{3}} \text { thickness } \\
\AA\end{array}$ & $\begin{array}{c}\text { PrA }^{*} \text { thickness } \\
\AA\end{array}$ \\
\hline S1 & $7.5 \pm 0.0 .3$ & $14.5 \pm 0.4$ \\
S2 & $22.9 \pm 0.3$ & $9.4 \pm 0.4$ \\
S3 & $9.3 \pm 0.3$ & $22.3 \pm 0.5$ \\
S4 & $18.9 \pm 0.8$ & $7.9 \pm 0.8$ \\
S5 & $5.6 \pm 0.3$ & $10.2 \pm 0.3$ \\
S6 & $13.1 \pm 0.3$ & $8.5 \pm 0.4$ \\
S7 & $10.0 \pm 0.3$ & $10.1 \pm 0.4$ \\
S8 & $5.7 \pm 0.3$ & $11.1 \pm 0.4$ \\
\hline
\end{tabular}

TABLE 5 Thicknesses of organic and biological layers, respectively $\mathrm{BS}^{3}$ and $\operatorname{Pr} \mathrm{A}^{*}$, linked to silane modified cSi surface. The values have been obtained by means of ellipsometric characterization.

is the value of $\Gamma$. We have repeated SE characterization after incubation with $\mathrm{BS}^{3}$ cross-linker and with PrA* Refractive indexes used for $\mathrm{BS}^{3}$-cross linker and $\operatorname{PrA}^{*}$ are 1.43 and 1.46 , respectively. $\mathrm{BS}^{3}$ refractive index is reported by reference [18]. PrA* refractive index has been calculated from Eq. (2) using the following values [19]: molar refractivity, $A=10173 \mathrm{~cm}^{3} / \mathrm{mol}$; molar volume, $V_{m}=37180 \mathrm{~cm}^{3} / \mathrm{mol}\left(V_{m}\right.$ is the product of molecular weight, $M_{w}=41000 \mathrm{~g} / \mathrm{mol}$ times the partial specific volume, $V_{p}=0.715 \mathrm{~cm}^{3} / \mathrm{g}$ ). Results of ellipsometric characterization are reported in Table 5.

The thickness of organic and biological films ranges between about 5 and $23 \AA$ and it does not seem to be correlated to silane thickness. From SE data, we can infer that more protein A can be bound to the surface in case of $30 \mathrm{~min}$ silanized chip (namely S1, S3, S5, S7) since the PrA* estimated thickness is always greater than $\mathrm{BS}^{3}$ layer thickness. When there is too much cross-linker, less protein can be found, probably due to steric hindrance of molecules (see values for S2, S4, S6). These considerations should be carefully trusted, since SE only gives a macroscopic average of layers thickness on an area of $1 \mathrm{~mm}^{2}$, which is not directly correlated to nanometric size of deposited films. We can certainly conclude that both organic and biological layers are of the same thickness order and 


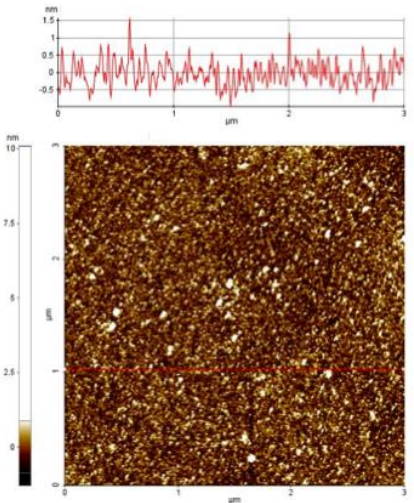

(s1)
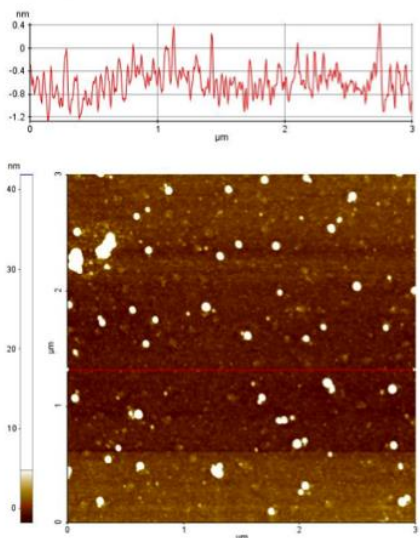

(s5)
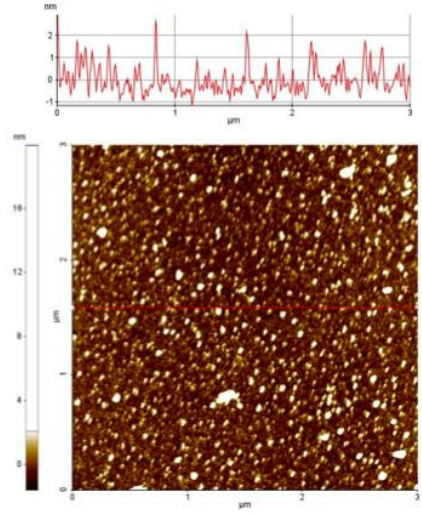

(\$2)
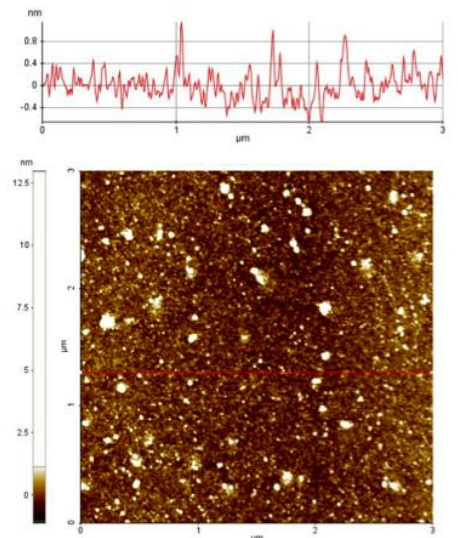

(S6)
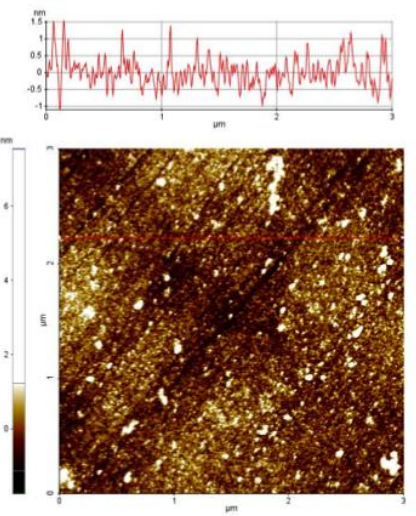

(s3)
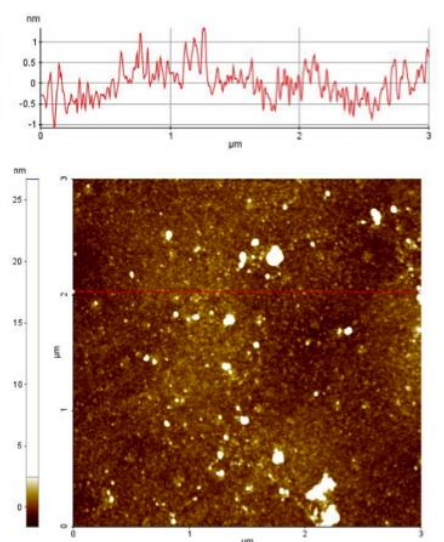

(s7)
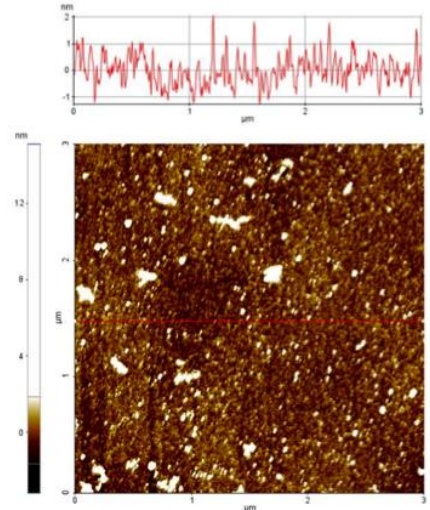

(s4)
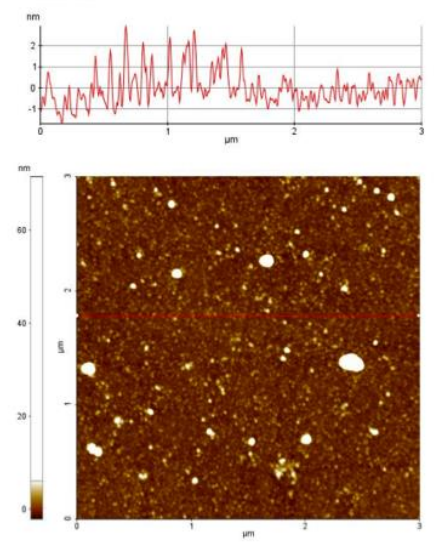

(s8)

FIG. $53 \mu \mathrm{m}$ wide AFM images of aminosilane modified CSi surfaces after PrA* functionalization.

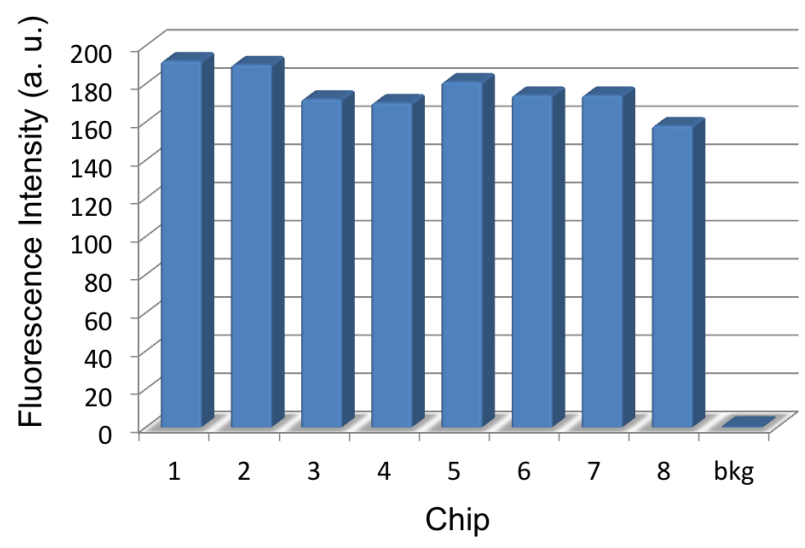

FIG. 4 Fluorescence intensity measured on the chips functionalized with $\operatorname{PrA}^{*}$ and on background (silane modified $\mathrm{CSi}$ surface $+\mathrm{BS}^{3}$ ).

that less cross-linker is better than too much. This conclusion is also supported by fluorescence data (Figure 4), summarized in details in Table 6. Fluorescence intensity is an integrated density calculated on an area of $100 \times 100 \mu \mathrm{m}^{2}$ using a freeware software, ImageJ. Each value reported in Table 6 is the average of three independent measurements on same sample. Fluorescence intensity of bare silicon dioxide is $(7 \pm 2)$ a. $u$.

The fluorescence intensity of odd samples (which has been incubated for $30 \mathrm{~min}$ ) is always greater than that of even ones

\begin{tabular}{|c|c|}
\hline Sample & $\begin{array}{c}\text { Fluorescence intensity } \\
\text { (a. u.) }\end{array}$ \\
\hline S1 & $192 \pm 2$ \\
S2 & $190 \pm 6$ \\
S3 & $172 \pm 1$ \\
S4 & $170 \pm 5$ \\
S5 & $181 \pm 2$ \\
S6 & $174 \pm 2$ \\
S7 & $174 \pm 2$ \\
S8 & $158 \pm 3$ \\
\hline
\end{tabular}

TABLE 6 Fluorescence intensity measured on the chips functionalized with PrA*. The values have been obtained as average of three different measurements on the same sample.

(incubated $60 \mathrm{~min}$ ), independently of considered silane. Anyway, absolute values of fluorescent intensities are very close each other, so that all samples have been effectively functionalized.

Quite differently, AFM images and characterization highlight some distinctions between PrA* surfaces on APTES and APDMES modified supports (see Figure 5). Quantitative measurements are expressed in terms of roughness, which values are reported for all samples in Table 7. Roughness of thermally grown silicon dioxide is $0.250 \mathrm{~nm}$. 


\begin{tabular}{|c|c|}
\hline Sample & $\begin{array}{c}\text { Roughness } \\
\text { (nm) }\end{array}$ \\
\hline S1 & 0.353 \\
S2 & 0.605 \\
S3 & 0.416 \\
S4 & 0.550 \\
S5 & 0.264 \\
S6 & 0.294 \\
S7 & 0.353 \\
S8 & 0.776 \\
\hline
\end{tabular}

TABLE 7 Root mean square roughness values of sample surfaces after $\operatorname{PrA}^{*}$ functionalization measured using an AFM $\left(3 \times 3 \mu \mathrm{m}^{3}\right)$

By examining both series (S1-S4; S5-S8) of number, it is evident that APDMES samples are smoother than APTES ones, except for sample S8 that is quite always very different from S5-S7. Moreover, in case of APTES samples, the thinner is the protein layer, the greater is the roughness, probably because of vertical inhomogeneity of APTES layer and PrA* film are very similar. APTES treated surfaces seem more crowded than APDMES ones, and again we believe that this is also due to self-assembling nature of APTES with respect to APDMES.

\section{CONCLUSIONS}

We have successfully functionalized silane modified flat oxidized silicon surfaces by ordinary chemical procedure, and we have studied how different solvents and incubation times can affect the quality of the protein layer on top. Quantitative measurements based on SE, WCA and AFM reveal that smoother and homogeneous film can be obtained using APDMES in toluene incubated for $30 \mathrm{~min}$. Anyway, all other samples show good functionalization degree as it can be seen by fluorescence characterization.

\section{ACKN OWLED GEMENTS}

Authors thank dr. G. Coppola of IMM-CNR for helpful discussions on AFM measurements. Work is partially supported by Italian National Operative Program PON01_02782.

\section{References}

[1] F. F. Bier, and S. Schumacher, "Integration in Bioanalysis: Technologies for Point-of-Care Testing," Adv. Biochem. Eng. Biotechnol. 133, 1-14 (2013).

[2] A. L. Washburn, and R. C. Bailey, "Photonics-on-a-chip: recent advances in integrated waveguides as enabling detection elements for real-world, lab-on-a-chip biosensing applications," Analyst 136, 227-236 (2011).

[3] S. Schumacher, J. Nestler, T. Otto, M. Wegener, E. EhrentreichFörster, D. Michel, K. Wunderlich, et al., "Highly-integrated lab-on-chip system for point-of-care multiparameter analysis," Lab. Chip. 12, 464-473 (2012).

[4] S.J. Attwood, A. M. C. Simpson, R. Stone, S. W. Hamaia, D. Roy, R. W. Farndale, M. Ouberai, and M. E. Welland, "A Simple Bioconjugate Attachment Protocol for Use in Single Molecule Force
Spectroscopy Experiments Based on Mixed Self-Assembled Monolayers," Int. J. Mol. Sci. 13, 13521-13541 (2012).

[5] V. K. Yadavalli, J. G. Forbes, and K. Wang, "Functionalized selfassembled monolayers on ultraflat gold as platforms for single molecule force spectroscopy and imaging," Langmuir 22, 6969-6976 (2006).

[6] Z. Yang, Y. Chevolot, T. Géhin, V. Dugas, N. Xanthopoulos, V. Laporte, T. Delair, et al., "Characterization of three aminofunctionalized surfaces and evaluation of antibody immobilization for the multiples detection of tumor markers involved in colorectal cancer," Langmuir 29, 1498-1509 (2013).

[7] R. K. DeLong, C. M. Reynolds, Y. Malcolm, A. Schaeffer, T. Severs, and A. Wanekays, "Functionalized gold nanoparticles for the binding, stabilization, and delivery of therapeutic DNA, RNA, and other biological macromolecules," Nanotechnol. Sci. Appl. 3, 53-63 (2013).

[8] J. J. Cooding, and S. Ciampi, "The molecular level modification of surfaces: From self-assembled monolayers to complex molecular assemblies," Chem. Soc. Rev. 40, 2704-2718 (2011).

[9] M. Zhu, M. Z. Lerum, and W. Chen, "How to prepare reproducible, homogeneous, and hydrolytically stable aminosilane-derived layers on silica," Langmuir 28, 416-423 (2013).

[10] N. Aissaoui, L. Bergaoui, J. Landoulsi, J. F. Lambert, and S. Boujday, "Silane layers on silicon surface: mechanism of interaction, stability, and influence on protein adsorption," Langmuir 28, 656-665 (2012).

[11] L. De Stefano, G. Oliviero, J. Amato, N. Borbone, G. Piccialli, L. Mayol, I. Rendina, et al. "Aminosilane functionalizations of mesoporous oxidized silicon for oligonucleotides synthesis and detection," J. R. Soc. Interface 10, 20130160 (2013).

[12] F. Zhang, K. Sautter, A. M. Larsen, D. A. Findley, R. C. Davis, H. Samha, and M. R. Lindford, "Chemical Vapor Deposition of three aminosilanes on silicon dioxide: surface characterization, stability, effects of silane concentration, and cyanine dye adsorption," Langmuir 26, 14648-14654 (2010).

[13] W. Kern, Handbook of semiconductor wafer cleaning technology: science, technology, and applications (William Andrew Publishing/Noyes, Norwich, 1993).

[14] Horiba Jobin Yvon DELTA PSI Software manual, ver. 2.4.3 158. (Horiba Scientific, 2011).

[15] E. D. Palik (ed.), Handbook of optical constants of solids (Academic Press, San Diego, 1985).

[16] P. Brocos, A. Piñeiro, R. Bravo, and A. Amigo, "Refractive indices, molar volumes and molar refractions of binary liquid mixtures: concepts and correlations," Phys. Chem. Chem. Phys. 5, 550-557 (2003).

[17] P. A. Cuypers, J. W. Corsel, M. P. Janssen, J. M. Kop, W. T. Hermens, and H. C. Hemker, "The adsorption of prothrombin to phosphatidylserine multilayers quantitated by ellipsometry," J. Biol. Chem. 258, 2426-2431 (1983).

[18] P. Déjardin (ed.), Proteins at solid-liquid interfaces (SpringerVerlag, Berlin, 2006).

[19] U. Jönsson, M. Malmqvist, I. Rönnberg, “Absorption of immunoglobulin $G$, protein $A$, and fibronectin in the submonolayer region evaluated by a combined Study of ellipsometry and radiotracer techniques," J. Colloid Interface Sci. 103, 360-372 (1985). 